\title{
Composição mineral e teor de ácido ascórbico nas folhas de quatro espé- cies olerícolas não-convencionais
}

\section{Danyela de Cássia da S Oliveira ${ }^{1}$; Carmen Wobeto ${ }^{2}$; Marcio R Zanuzo ${ }^{2}$; Cristiane Severgnini}

${ }^{1}$ UFPel, Depto. Fitotecnia, C. Postal 354, 96001-970 Pelotas-RS; danyeladecassia@gmail.com; ${ }^{2}$ UFMT, Campus de Sinop, Av. Alexandre Ferronato 1200, Setor Industrial, 78557-267 Sinop-MT; wobeto2003@yahoo.com.br (*autor para correspondência); zanuzo@yahoo.com; cristianesevergnini@gmail.com

\section{RESUMO}

As hortaliças não-convencionais apresentam maiores teores de nutrientes do que as convencionais, contudo seu potencial de produção e consumo é negligenciado, havendo poucas informações sobre a composição química destas espécies. Neste trabalho determinaram-se os teores de ácido ascórbico (vitamina C) e de minerais das seguintes hortaliças folhosas não-convencionais: taioba, ora-pro-nobis, bertalha e beldroega. As folhas de taioba apresentaram os maiores teores de ácido ascórbico (198,33 mg $100 \mathrm{~g}^{-1}$ de matéria fresca) e

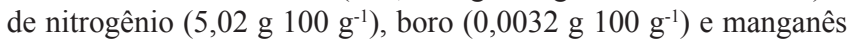
$\left(0,007 \mathrm{~g} 100 \mathrm{~g}^{-1}\right)$, na matéria seca (MS). As folhas da ora-pro-nobis apresentaram teores similares de ácido ascórbico e os maiores de fósforo $\left(0,45 \mathrm{~g} 100 \mathrm{~g}^{-1} \mathrm{MS}\right)$ e de magnésio $\left(0,68 \mathrm{~g} 100 \mathrm{~g}^{-1} \mathrm{MS}\right)$. Nas folhas da beldroega verificaram-se o maiores teores de ferro $(0,032$ g $\left.100 \mathrm{~g}^{-1} \mathrm{MS}\right)$ e de cálcio (2,39 g $\left.100 \mathrm{~g}^{-1} \mathrm{MS}\right)$. Quanto aos níveis de zinco as espécies que se destacaram foram a bertalha $(0,0057 \mathrm{~g} 100$ $\left.\mathrm{g}^{-1} \mathrm{MS}\right)$ e a ora-pro-nobis $\left(0,0059 \mathrm{~g} 100 \mathrm{~g}^{-1} \mathrm{MS}\right)$. Não houve diferença significativa entre as espécies com relação ao nível de potássio $(3,71$ a 3,87 g $\left.100 \mathrm{~g}^{-1} \mathrm{MS}\right)$. Verificou-se que as espécies estudadas poderão se tornar boas fontes de acido ascórbico e minerais com a difusão de seu uso pela população.

Palavras-chave: Portulaca oleracea, Xanthosoma sagittifolium, Pereskia aculiata, Basella rubra, beldroega, taioba, ora-pro-nobis, bertalha, hortaliças não-convencionais.

\section{ABSTRACT}

Mineral composition and ascorbic acid content in four nonconventional leafy vegetables species

Non-conventional vegetables show higher nutrient levels than the conventional ones, but their potential for production and consumption has been neglected and there have been few studies about their chemical composition. This study has investigated the levels of ascorbic acid (vitamin C) and minerals in the following non-conventional leafy vegetables: taro, ora-pro-nobis, bertalha and purslane. The taro leaves showed the highest levels of ascorbic

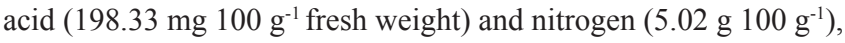

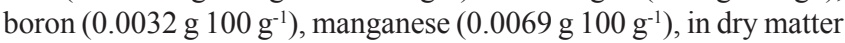
(DM). The leaves of ora-pro-nobis showed similar levels of ascorbic

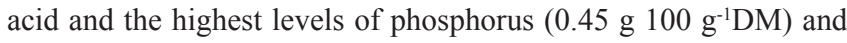

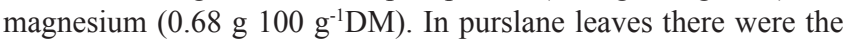

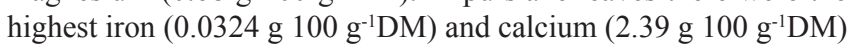
content. Concerning to zinc levels, the species that stood out were

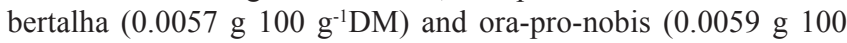
$\left.\mathrm{g}^{-1} \mathrm{DM}\right)$. As for potassium, there was no significant difference amongst

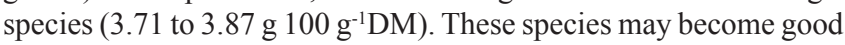
sources of ascorbic acid and minerals with the spread of their use by the population.

Keywords: Portulaca oleracea, Xanthosoma sagittifolium, Pereskia aculiata, Basella rubra, purslane, taro, ora-pro-nobis, bertalha, nonconventional vegetables.

\section{(Recebido para publicação em 5 de setembro de 2012; Aceito em 16 de agosto de 2013)} (Received on September 5, 2012; accepted on August 16, 2013)

\begin{abstract}
$\mathrm{A}$ s hortaliças associam duas condições desejáveis para uma alimentação saudável: baixo conteúdo de calorias e bons teores de vitaminas e minerais, conforme pode ser constatado nas tabelas nutricionais de Franco (2004) e NEPA/UNICAMP (2011).

As hortaliças não-convencionais são aquelas que não estão organizadas em cadeias produtivas e por isso não despertam o interesse comercial das empresas de sementes, fertilizantes ou agroquímicos. Sua utilização é geralmente restrita a determinadas regiões e com inserção na culinária e na cultura
\end{abstract}

destes locais, como é o caso de ora-pro-nobis em Sabará-MG, onde é realizado o festival anual desta hortaliça (MAPA, 2010).

Apesar destas espécies terem seu cultivo negligenciado, vários estudos relatam que elas apresentam maiores teores de vitamina $\mathrm{C}$ e minerais que as convencionalmente empregadas, como por exemplo, repolho, alface e couve (Gupta et al., 2005; Flyman \& Afolayan, 2006; Kinupp \& Barros, 2008; Uusiku et al., 2010; Schönfeldt \& Pretorius, 2011; Andarwulan et al., 2012).

Os dados sobre a composição quí- mica das hortaliças, objeto deste trabalho, ainda são escassos. Para a taioba, cultivada em regiões de Minas Gerais e Espírito Santo, já foi atestado seu potencial nutricional (Pinto et al., 1999; Morais et al., 2006). Contudo, não há estudos sobre seus teores de vitamina C e minerais quando cultivada no estado do Mato Grosso, uma vez que as condições edafoclimáticas podem afetar a composição química e nutracêutica das hortaliças (Lee \& Kader, 2000).

As folhas de ora-pro-nobis são reconhecidas por apresentarem altos teores de proteínas (Kinupp \& Barros, 2008), 
porém não há registros de seus níveis de minerais e vitaminas. Já, a beldroega é reconhecida como planta invasora (Erasmo et al., 2004) e seu uso culinário é negligenciado no Brasil, apesar de ser utilizada na Europa e África (Odhav et al., 2007; Uusiku et al., 2010; Andarwulan et al., 2012). Além disso, não há registros sobre os teores de vitamina $\mathrm{C}$ das folhas de bertalha, sendo somente relatados seus níveis de zinco (Andrade et al., 2005).

Neste trabalho determinaram-se os teores de ácido ascórbico e dos minerais, N, P, K, Ca, Mg, S, B, Cu, $\mathrm{Fe}, \mathrm{Mn}$ e $\mathrm{Zn}$, das seguintes hortaliças folhosas: taioba, ora-pro-nobis, bertalha e beldroega, ainda pouco cultivadas em plantios comerciais e utilizadas para a alimentação humana.

\section{MATERIAL E MÉTODOS}

Os rizomas da taioba (Xanthosoma sagittifolium), o caule usado para propagação vegetativa da ora-pro-nobis (Pereskia aculiata), as sementes da beldroega (Portulaca oleracea) e da bertalha (Basella rubra) foram obtidos da horta experimental da Universidade do Estado de Mato Grosso, Campus de Cáceres.

Os materiais utilizados para a propagação das culturas foram transportados até a área experimental da Universidade Federal de Mato Grosso, Campus de Sinop, em julho de 2008, onde foram realizados o plantio, tratos culturais e colheita.

A ora-pro-nobis foi propagada pelo método da estaquia, empregando-se um pedaço do caule da região intermediária, localizada entre as partes mais tenras e as mais lenhosas da haste. Esse procedimento foi realizado a partir do pré-plantio de mudas e aos 90 dias realizou-se o corte de estacas com aproximadamente $5 \mathrm{~cm}$. As estacas foram colocadas em sacolas plásticas de $750 \mathrm{~mL}$ com substrato contendo esterco bovino, areia e casca de arroz carbonizada (1:2:1, v:v:v). O material foi mantido sob sombrite $50 \%$, até o desenvolvimento das gemas iniciais, o que permitiu seu transplante.

Os rizomas de taioba foram plantados em sacos plásticos com capacidade de $750 \mathrm{~mL}$ no substrato indicado acima. As sementes de beldroega e bertalha foram semeadas em bandejas de isopor sobre o substrato comercial Plantmax ${ }^{\circledR}$.

O plantio das mudas de taioba, ora-pro-nobis, bertalha e beldroega foi realizado nos canteiros definitivos com espaçamento de $80 \times 80 ; 60 \times 60 ; 40$ x 40 e 30 x $30 \mathrm{~cm}$, respectivamente, e cultivadas por 120 dias, empregando-se adubação orgânica e química com aplicação, nas covas, de $2 \mathrm{~kg} \mathrm{ha}^{-1}$ de cama de aviário e $50 \mathrm{~g} \mathrm{cova}^{-1}$ de adubo NPK (4-14-8).

A área de plantio foi previamente corrigida utilizando-se calcário dolomítico como fonte de $\mathrm{Ca}$ e $\mathrm{Mg}$ na quantidade de 2,0 $\mathrm{t} \mathrm{ha}^{-1}$. Os canteiros foram delineados utilizando-se enxada rotativa com largura de 1,20 m. A irrigação foi realizada com o uso de fitas gotejadoras conforme a evapotranspiração do tanque classe A. Tanto os tratos culturais como o controle de plantas daninhas foram realizados por meio da capina manual. Os insetos foram controlados por catação manual e uso de armadilhas.

As folhas maduras, completamente expandidas, sem pecíolos e sem sintomas de amarelecimento foram coletadas em sacolas de papel e divididas quanto ao procedimento das análises. As folhas para as análises de umidade e de ácido ascórbico foram coletadas e imediatamente submetidas aos procedimentos analíticos, realizados em laboratório da Universidade Federal de Mato Grosso, Campus de Sinop. A análise de umidade foi realizada através de método gravimétrico (AOAC, 1995) e para determinação do teor de acido ascórbico empregou-se o método espectrofotométrico descrito por Stroehecker \& Henning (1967).

As folhas a serem usadas para as análises mineralógicas foram submetidas a secagem em estufa de circulação forçada de ar a $70^{\circ} \mathrm{C}$, por três dias. Após este período o material foi triturado em moinho tipo Willey, armazenado em frasco âmbar e enviado para o Laboratório de Análise Foliar da Universidade Federal de Lavras, onde foram determinados seus teores de ferro, zinco, manganês, cobre, magnésio e cálcio, por espectrofotometria de absorção atômica. O teor de potássio foi determinado por fotometria de chama (Malavolta et al., 1997) e do fósforo e enxofre por colorimetria (AOAC, 1995).

O delineamento estatístico empregado foi inteiramente casualizado, com 7 repetições para cada tratamento (espécie). Os dados obtidos foram submetidos à análise de variância e as médias comparadas pelo teste de Tukey $(\mathrm{p} \leq 0,05)$.

\section{RESULTADOS E DISCUSSÃO}

Na Tabela 1 estão apresentadas as famílias botânicas e as espécies de hortaliças folhosas analisadas e seus respectivos teores médios de umidade $\mathrm{e}$ ácido ascórbico. Observou-se variação de umidade das folhas entre as espécies

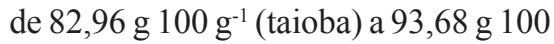

Tabela 1. Famílias e espécies de hortaliças não convencionais e seus respectivos teores médios de umidade e ácido ascórbico na matéria fresca das folhas (families and species of non-conventional vegetables and their respective average content of moisture and ascorbic acid in fresh weight basis of leaves). Sinop, UFMT, 2009.

\begin{tabular}{lcccc}
\hline Família & Espécie & Nome comum & Umidade (g/100 g) & Ácido ascórbico (mg/100 g) \\
\hline Araceae & Xanthosoma sagittifolium & Taioba & $82,96 \mathrm{c}^{*}$ & $198,33 \mathrm{a}$ \\
Cactaceae & Pereskia aculeata & Ora-pro-nobis & $86,99 \mathrm{~b}$ & $192,21 \mathrm{a}$ \\
Basellaceae & Basella rubra & Bertalha & $92,92 \mathrm{a}$ & $139,56 \mathrm{~b}$ \\
Portulacaceae & Portulaca oleracea & Beldroega & $93,68 \mathrm{a}$ & $38,56 \mathrm{c}$ \\
\hline
\end{tabular}

*Médias seguidas de mesma letra nas colunas, não diferem entre si pelo teste de Tukey $(\mathrm{p} \leq 0,05)$ (means followed by the same letter within a column are not significantly different, Tukey, $\mathrm{p} \leq 0,05$ ). 
Tabela 2. Teores médios de minerais (g/100 g MS) nas folhas de hortaliças não-convencionais (average levels of minerals (g/100 g dry matter), in the leaves of non-conventional vegetables). Sinop, UFMT, 2009.

\begin{tabular}{lccccc}
\hline Minerais & Taioba & Ora-pro-nobis & Bertalha & Beldroega & CV (\%) \\
\hline $\mathrm{N}$ & $5,02 \mathrm{a}^{*}$ & $3,47 \mathrm{~b}$ & $2,93 \mathrm{c}$ & $2,17 \mathrm{~d}$ & 8,51 \\
$\mathrm{P}$ & $0,37 \mathrm{~b}$ & $0,45 \mathrm{a}$ & $0,35 \mathrm{~b}$ & $0,35 \mathrm{~b}$ & 8,13 \\
$\mathrm{~K}$ & $3,82 \mathrm{a}$ & $3,74 \mathrm{a}$ & $3,87 \mathrm{a}$ & $3,71 \mathrm{a}$ & 3,17 \\
$\mathrm{Ca}$ & $2,39 \mathrm{a}$ & $2,16 \mathrm{~b}$ & $2,05 \mathrm{~b}$ & $2,39 \mathrm{a}$ & 3,96 \\
$\mathrm{Mg}$ & $0,31 \mathrm{~d}$ & $0,68 \mathrm{a}$ & $0,52 \mathrm{c}$ & $0,58 \mathrm{~b}$ & 2,50 \\
$\mathrm{~S}$ & $0,28 \mathrm{c}$ & $0,36 \mathrm{~b}$ & $0,52 \mathrm{a}$ & $0,20 \mathrm{~d}$ & 5,85 \\
$\mathrm{~B}$ & $0,0032 \mathrm{a}$ & $0,0028 \mathrm{~b}$ & $0,0024 \mathrm{c}$ & $0,0028 \mathrm{~b}$ & 7,45 \\
$\mathrm{Cu}$ & $0,0012 \mathrm{a}$ & $0,0009 \mathrm{~b}$ & $0,0005 \mathrm{c}$ & $0,0011 \mathrm{ab}$ & 8,94 \\
$\mathrm{Mn}$ & $0,0069 \mathrm{a}$ & $0,0028 \mathrm{~d}$ & $0,0042 \mathrm{c}$ & $0,0058 \mathrm{~b}$ & 3,60 \\
$\mathrm{Zn}$ & $0,0033 \mathrm{~b}$ & $0,0059 \mathrm{a}$ & $0,0057 \mathrm{a}$ & $0,0020 \mathrm{c}$ & 4,89 \\
$\mathrm{Fe}$ & $0,0081 \mathrm{c}$ & $0,0094 \mathrm{c}$ & $0,0213 \mathrm{~b}$ & $0,0324 \mathrm{a}$ & 5,41 \\
\hline
\end{tabular}

*Médias seguidas de mesma letra nas linhas, não diferem entre si pelo teste de Tukey $(\mathrm{p} \leq 0,05)$ (means followed by the same letter within rows are not significantly different, Tukey, $\mathrm{p} \leq 0,05$ ).

$\mathrm{g}^{-1}$ (beldroega).

As folhas de taioba e ora-pro-nobis apresentaram os maiores teores de ácido ascórbico. O teor desta vitamina corrobora com os relatados de Morais et al. (2006) que avaliaram o efeito do tipo de cultivo da taioba (natural, orgânico e convencional) nos teores de vitamina $C$ de suas folhas e relataram variação de 119,67 a 223,00 mg $100 \mathrm{~g}^{-1}$ de materia fresca (MF). As folhas de beldroega apresentaram teores de ácido ascórbico

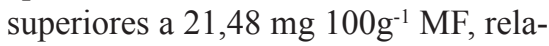
tados por Andarwulan et al. (2012) para esta espécie, quando cultivada na Indonésia. As diferenças observadas devem ser decorrentes da diversidade genética e das condições edafoclimáticas.

Comparando-se o nível de variação no teor de ácido ascórbico nas folhas das espécies analisadas com outras hortaliças folhosas com cultivo comercial difundido, como a couve manteiga e a rúcula, que apresentam teores de 96,7 mg $100 \mathrm{~g}^{-1}$ MF e de 46,3 mg $100 \mathrm{~g}^{-1}$ MF, respectivamente, constata-se que somente a beldrogeda apresentou teores menores, indicando que as folhas de taioba, bertalha ou ora-pro-nobis são boas alternativas para suprir as necessidades diárias desta vitamina (NEPA/ UNICAMP, 2011).

Outros estudos também destacaram teores de vitamina $\mathrm{C}$ elevados em hortaliças folhosas não convencionais, como por exemplo, para Amaranthus sp. e
Vernonia sp., com variação de 46 a 126 e de 51 a $198 \mathrm{mg} 100 \mathrm{~g}^{-1} \mathrm{MF}$, respectivamente (Steyn et al., 2001; Ejoh et al., 2007; Uusiku et al., 2010).

Na Tabela 2 são mostrados os teores médios de minerais nas folhas das espécies investigadas. As folhas de taioba apresentaram os maiores teores de nitrogênio, cálcio, boro, cobre e manganês, enquanto nas folhas de ora-pro-nobis foram observados os maiores níveis de fósforo, magnésio e zinco. As folhas de bertalha apresentaram os maiores teores de enxofre e as de beldroega se destacaram pelos maiores níveis de ferro. Observa-se também que os teores de potássio não apresentaram diferenças significativas entre as espécies estudadas.

Os níveis de nitrogênio nas folhas de taioba e ora-pro-nobis são indicativos do bom teor protéico destas duas espécies. Vieira (1983) destacou a possibilidade do uso da ora-pro-nobis como fonte de proteína foliar na alimentação humana.

Os níveis de $\mathrm{Ca}, \mathrm{P}, \mathrm{Zn}, \mathrm{Cu}, \mathrm{Mg}$ e $\mathrm{K}$ nas folhas de taioba foram similares aos descritos por Pinto et al. (1999), enquanto os teores de $\mathrm{Fe}$ e $\mathrm{Mn}$ foram inferiores aos relatados por estes autores, ou seja $237,4 \mathrm{mg} \mathrm{kg}^{-1} \mathrm{MS}$ e $107,6 \mathrm{mg} \mathrm{kg}^{-1} \mathrm{MS}$, respectivamente. Nas folhas de bertalha, os teores de zinco foram similares aos descritos por Andrade et al. (2005). Odhav et al. (2007) indicaram para a beldroega teores de $\mathrm{Fe}$ (42 mg $100 \mathrm{~g}^{-1}$
MS) e de Zn (34 mg $\left.100 \mathrm{~g}^{-1} \mathrm{MS}\right)$, maiores que os observados neste trabalho. As características genéticas das cultivares de uma mesma espécie e a composição química do solo devem ter influenciado as diferenças observadas.

Comparando-se os níveis dos minerais $\mathrm{P}, \mathrm{K}, \mathrm{Ca}$ e $\mathrm{Mg}$ nas hortaliças estudadas (Tabela 2), com hortaliças folhosas como chicória $(0,26 \mathrm{~g} 100$ $\left.\mathrm{g}^{-1} \mathrm{MS}\right)$, repolho (2,83 g $\left.100 \mathrm{~g}^{-1} \mathrm{MS}\right)$, agrião (2,13 g $\left.100 \mathrm{~g}^{-1} \mathrm{MS}\right)$ e couve man-

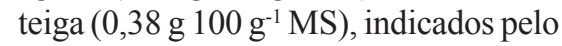
NEPA/UNICAMP (2011), constata-se que as espécies analisadas são boas fontes destes minerais, pois apresentam teores superiores aos relatados para as cultivadas comercialmente.

Quanto aos níveis de enxofre, as folhas de ora-pro-nobis e bertalha apresentaram teores maiores que os relatados para a couve $\left(0,31{\mathrm{~g} 100 \mathrm{~g}^{-1}}^{-1}\right.$ MS) e o repolho (0,32 g $\left.100 \mathrm{~g}^{-1} \mathrm{MS}\right)$, por Franco (2004). Vale ressaltar que segundo Mahan \& Escott-Stump (1998), o enxofre é integrante dos aminoácidos metionina, cistina e cisteína, e pode fornecer indicações dos níveis destes aminoácidos nos alimentos.

Os teores dos minerais nas hortaliças estudadas (Tabela 2) também são comparáveis aos encontrados em outras espécies também não convencionalmente utilizadas, Muehlenbeckia sagittifolia, Chamissoa altissima, Amaranthus sp e Cleome $s p$, as quais apresentaram 
destaque, respectivamente, para os níveis de $\mathrm{P}\left(0,35 \mathrm{~g} 100 \mathrm{~g}^{-1} \mathrm{MS}\right), \mathrm{K}(4,1$

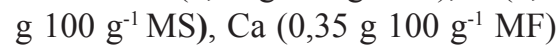
e de $\mathrm{Mg}\left(0,06 \mathrm{~g} 100 \mathrm{~g}^{-1} \mathrm{MF}\right)$, enquanto nas folhas de Aystasia gangetica foram relatos níveis apreciáveis de $\mathrm{Zn}(0,0078$ g $\left.100 \mathrm{~g}^{-1} \mathrm{MF}\right)$ e de Fe $\left(0,015{\mathrm{~g} 100 \mathrm{~g}^{-1}}^{-}\right.$ MF), por Gupta et al. (2005), Odhav et al.( 2007), Kinupp \& Barros (2008) e Uusiku et al. (2010).

A beldroega é uma boa fonte de ferro, pois como base nas exigências nutricionais deste mineral para crianças, $10 \mathrm{mg} /$ dia (NRC, 1989) e nos teores apresentados por esta espécie, 32,4 mg $100 \mathrm{~g}^{-1} \mathrm{MS}$, constata-se que $100 \mathrm{~g}$ de folhas de beldroega seriam suficientes para suprir parte das necessidades diárias deste mineral. Isto indica que o uso das folhas desta espécie é uma alternativa para amenizar a anemia provocada pela deficiência de ferro, que é a mais comum das carências nutricionais (Jordão et al., 2009).

Baseando-se nas recomendações médias para adultos, independente do sexo, de fósforo (1.000 mg/dia), potássio (2.000 mg/dia), magnésio (330 $\mathrm{mg} / \mathrm{dia})$, cálcio (1.000 mg/dia), cobre (0,35 mg/dia) e manganês $(3,5 \mathrm{mg} /$ dia), verifica-se que porções de $100 \mathrm{~g}$ de folhas frescas de ora-pro-nobis, bertalha e beldroega, supririam respectivamente $58 \%, 14 \%$ e $11 \%$ destas necessidades diárias. A mesma quantidade de folhas de taioba seria suficiente para fornecer 41,57 e 34\% das exigências de $\mathrm{Ca}$, $\mathrm{Cu}$ e Mn, respectivamente. A inclusão destas espécies no cardápio pode auxiliar na redução das carências nutricionais de populações (NRC, 1989; WHO/FAO, 2004).

Conclui-se que as hortaliças não-convencionais, taioba, ora-pro-nobis, bertalha e beldroega apresentam altos níveis de ácido ascórbico e de minerais, indicando a possibilidade de inserção das mesmas na cadeia produtiva e sua difusão como ingrediente de dietas alimentares.

\section{AGRADECIMENTOS}

\section{À FAPEMAT pelo suporte finan-} ceiro.

\section{REFERÊNCIAS}

ANDARWULAN N; KURNIASIH D; APRIADY RA; RAHMAT H; ROTO AV; BOLLING, BW. 2012. Polyphenols, carotenoids, and ascorbic acid in underutilized medicinal vegetables. Journal of Functional Foods 4: 339-347.

ANDRADE ECB; TEODORO AJ; TAKASE I. 2005. Determinação dos teores de zinco em diferentes extratos de hortaliças dos tipos A e B. Ciência e Tecnologia de Alimentos 25: 265-270.

AOAC - ASSOCIATION OF OFFICIAL ANALYTICAL CHEMISTS. 1995. Official methods of analysis of the Association of the Analytical Chemists. 16 ed. Gaithersburg, MD: AOAC. $1015 \mathrm{p}$.

EJOH RA; NKONGA DV; INOCENT G; MOSES MC. 2007. Nutritional components of some non-conventinal leafy vegetables consumed in Cameroon. Pakistan Journal of Nutrition 6:712-717.

ERASMO EAL; PINHEIRO LLA; COSTA NV. 2004. Levantamento fitossociológico das comunidades de plantas infestantes em áreas de produção de arroz irrigado cultivado sob diferentes sistemas de manejo. Planta Daninha 22: 195-201.

FLYMAN MV; AFOLAYAN AJ. 2006. The suitability of wild vegetables for alleviating human dietary deficiencies. South African Journal of Botany. 72: 492-497.

FRANCO G. 2004. Tabela de composição química dos alimentos. 9 ed. São Paulo: Atheneu. 307 p.

GUPTA S; LAKSHMI AJ; MANJUNATH MN; PRAKASH J. 2005. Analysis of nutrient and antinutrient content of underutilized green leafy vegetables. LWT-Food Science and Technology 38: 339-345.

JORDÃO RE; BERNARDI JLD, BARROS FILHO AA. 2009. Prevalência de anemia ferropriva no Brasil: uma revisão sistemática. Revista Paulista de Pediatria, 27:9098.

KINUPP VF; BARROS IBI. 2008. Teores de proteína e minerais de espécies nativas, potenciais hortaliças e frutas. Ciências e Tecnologia de Alimentos 28: 846-857.

LEE SK; KADER AA. 2000. Preharvest and postharvet factors influencing vitamin $\mathrm{C}$ content of horticultural crops. Postharvest Biology \& Technology 20: 207-220.

MAHAN LK; ESCOTT-STUMP S. 1998. Krause: alimentos, nutrição e dietoterapia. 9. ed. São Paulo: Roca. 1179 p.

MALAVOLTA E; VITTI GC; OLIVEIRA SA.
1997. Avaliação do estado nutricional das plantas. Piracicaba: Potafos. 319 p.

MAPA - Ministério da Agricultura, Pecuária e Abastecimento, 2010. Manual de hortaliças não-convencionais. Brasília: MAPA/ACS, 92 p. Disponível em http://www.agricultura. gov.br/arq_editor/file/vegetal/Qualidade/ Qualidade $\% 20$ dos $\% 20$ Alimentos $/$ manual $\% 20$ hortali\%C3\%A7as_WEB_F.pdf. Acessado em 10 de fevereiro de 2012 .

MORAIS VS; MARTINS JA; WEBER MB; SENA DR. 2006. Efeito do tipo de cultivo no conteúdo de vitamina $\mathrm{C}$ em folhas de taioba (Xanthosoma sagittifolium Schoot). Revista Capixaba de Ciência e Tecnologia 1: 64-68.

NEPA/UNICAMP. 2011. Tabela brasileira de composição de alimentos - TACO. 4 ed. Disponível em http://www.unicamp.br/nepa/ taco/tabela.php?ativo=tabela. Acessado em 31 de maio de 2011.

NRC - NATIONAL RESEARCH COUNCIL. 1989. Recommended dietary allowances. 10 ed. Washington: National Academic. 284p.

ODHAV B; BEEKRUM S; AKULA US; BAIJNATH, H. 2007. Preliminary assessment of nutritional value of traditional leafy vegetables in KwaZulu-Natal, South Africa. Journal of Food Composition and Analysis 20: 430-435.

PINTO NAVD; CARVALHO VD; BOAS BMV. 1999. Caracterização mineral das folhas de taioba. Ciência e Agrotecnologia 23: 57-61.

SCHÖNFELDT HC; PRETORIUS B. 2011. The nutrient content of five traditional South African dark green leafy vegetables - A preliminary study. Journal of Food Composition and Analysis 24: 1141-1146.

STEYN NP; OLIVIER J; WINTER P; BURGER S; NESAMVUNI C. 2001. A survey of wild green leafy vegetables and their potential in combating micronutrient deficiencies in rural populations. South African Journal of Science 97: 276-278.

STROEHECKER R; HENNING HM. 1967. Analises de vitaminas: métodos comprobados. Madrid: Paz Montalvo. 428 p.

UUSIKU NP; OELOFSE A; DUODU KG; BESTER MJ; FABER M. 2010. Nutritional value of leafy vegetables of Sub-saharan Africa and their potential contribution to human health: A review. Journal of Food Composition and Analysis 23: 499-509.

VIEIRA EC. 1983. Leaf protein research in Brazil. In: TELEK L; GRAHAM HD (eds). Leaf protein concentrates. Westport: AVI Publishing Co. p. 661-668.

WHO/FAO - World Health Organization and Food and Agriculture Organization of the United Nations. 2004. Vitamin and mineral requirements in human nutrition. 2 ed. Rome: World Health Organization. 341 p. Disponível em http://whqlibdoc.who.int/ publications/2004/9241546123.pdf. Acessado em 20 de março de 2012. 